\title{
Forschungsnotiz
}

\section{Gemeinsamkeiten und Unterschiede von Konservatismus und Autoritarismus}

\section{Josef Schenk}

Institut für Psychologie der Universität Würzburg

Ludwigstraße 6, D-8700 Würzburg

\section{Problemlage}

Neben Politologie, Geschichtswissenschaft und Soziologie hat sich auch die Sozialpsychologie mit Konservatismus und Faschismus beschäftigt. Sie tat dies, indem sie entsprechende Einstellungen via Skalen zu messen versuchte und Korrelate zu diesen Einstellungen aufzeigte.

Die Beschreibung konservativer Einstellung reicht bis in die 30er Jahre zurück (z.B. Carlson 1934, Ferguson 1939) und wurde bis in die unmittelbar zurückliegende Vergangenheit fortgesetzt (z.B. Eysenck 1954, 1975, Wilson/Patterson 1968, Siddiqui/Jansen/Haara 1971, Schneider/Minkmar 1972, Schenk 1980). Die theoretische Fassung der konservativen Haltung wurde im englischsprachigen Bereich vor allem von Burke (dt. Ausgabe 1967), im deutschsprachigen Raum u.a. von Mannheim (1927), Merkatz (1957) und Mühlenfeld (1952) geleistet. In den einzelnen Skalen wird das Konzept Konservatismus mehr oder weniger weit gefaßt, wesentliche Themen sind Tradition und Evolution, Wirtschaftsordnung, Einstellung zur Autorität, moralische Haltung, Individualität, nationale Eigenständigkeit, nationale Sicherheit, Behandlung von Verbrechern, Beziehung der Geschlechter.

Während die Beschreibung der konservativen Haltung mehr oder weniger eng an dem Selbstverständnis konservativer Theoretiker orientiert ist, ist die Deskription der faschistischen bzw. autoritären Einstellung stark durch die Psychoanalyse geprägt. Die entscheidende Arbeit stammt von Adorno et al. (1950); nach ihnen ist der tendenziell faschistische bzw. autoritäre Mensch durch Unterwürfigkeit gegenüber Autoritäten, Konventionalität, Verleugnung eigener Gefuihle, Denken in Machtkategorien ausgezeichnet. Deutsche Fassungen der F-Skala wurden von Reghmann (1966) und Freyhold (1971) entwickelt.
Die empirische Fassung von Konservatismus und Faschismus bzw. Autoritarismus via Skalen erfolgte nach unterschiedlichen Konzeptionen, es soll in jedem Fall jedoch rechtsgerichtete weltanschauliche Haltung gemessen werden. Wurde damit letztlich doch nur das gleiche aus unterschiedlichen Perspektiven (Selbstverständnis Konservativer bzw. psychoanalytische Konzeption) gemessen? Wilson/Patterson (1968), Ray (1973) und Thomas (1975) setzen die beiden Konzepte nahezu gleich, für Adorno et al. (1950) ist konservative Einstellung nur ein indirektes $\mathrm{Maß}$ für autoritäre Tendenzen. Adorno et al. und andere Autoren (Rokeach 1960; Kohn 1972, 1974) fanden Korrelationen in der GröBenordnung von $\mathrm{r}=.50$ zwischen beiden Einstellungen.

Für eine Gleichsetzung beider Einstellungen spricht, daß sowohl bei der konservativen wie bei der autoritären Haltung ein Mißtrauen gegenüber den Fähigkeiten des einzelnen deutlich wird. Daneben gibt es jedoch möglicherweise auch signifikante Unterschiede zwischen den beiden Einstellungen. Autoritarismus und Anomie korrelieren signifikant miteinander (Srole 1950; Roberts/Rokeach 1956; McDill 1962; Struening/ Richardson 1965); der Autoritäre findet nicht die erwarteten einfachen und strikten Normen in der Gesellschaft vor und fühlt sich auf Grund seiner unterdrückenden Erziehung relativ hilflos. Ganz anders hingegen der Konservative: er ist in seiner sozialen Welt - solange diese Verwurzelung bei stabilen Verhältnissen gelingt - fest verankert und deshalb auch ideologielos (Mühlenfeld 1952). Erst die Gefährdung der gewordenen Ordnung durch Ideologien zwingt den Konservativen zur Formulierung eigener konservativer Prinzipien (Huntington 1957). Man könnte daher erwarten, daß ein Konservativer in einer relativ stabilen Welt wenig anomisch ist. Neben Gemeinsamkeiten zwischen Konservativen und Autoritären müßte es demnach auch signifikante Unterschiede geben. 


\section{Fragestellung}

Im folgenden soll deshalb untersucht werden, $o b$

- sich der positive Zusammenhang zwischen Konservativismus und Autoritarismus reproduzieren läßt,

- sich dieser eventuelle positive Zusammenhang durch eine beiden Einstellungen gemeinsame strikte moralische Haltung erklären läßt,

- neben Gemeinsamkeiten auch Unterschiede nachweisbar sind, die durch die Variable Anomie bestimmt sind.

\section{Methodik}

\subsection{Die Testbatterie}

Autoritarismus wird gemessen durch die A-Skala von Freyhold (1971); die A-Skala ist eine deutliche Version der amerikanischen F-Skala und mißt autoritäre Einstellung.

Konservatismus wird gemessen durch die Konservatismus-Skala von Schenk (1980). Die Skala wurde faktorenanalytisch entwickelt und erwies sich über mehrere Stichproben hinweg als stabile Dimension. Ihre Inhalte sind: Kapitalismus vs. Sozialismus, Reformen vs. Revolution, Einstellung zur politischen Autorität, Bedeutung von Tradition, Art der Friedenspolitik, Bekämpfung von Verbrechen. Beispiele für Items aus dieser Skala: ,Jeder Vertreter des Staates hat einen gewissen Anspruch auf Vertrauen von seiten der Staatsbürger". "In einem kapitalistischen Land hat jeder eine gute Chance, reich zu werden.“ „Nur Reformen, die das Bestehende nicht radikal zerstören, sondern es schrittweise verändern, können den sicheren und beständigen Fortschritt unseres Volkes garantieren".

Beide Skalen haben eine gute interne Konsistenz (s. Schenk 1979, 1980). Zur Erklärung der möglichen Gemeinsamkeit zwischen beiden Einstellungen wurde vom Autor eine Skala zur Messung rigoroser moralischer Prinzipien entwickelt. Die Skala sollte eine Haltung erfassen, die man als konsequent, sogar als unerbittlich kennzeichnen kann. Die Bedeutung von Prinzipien wird in ihr hervorgehoben, Fehler werden nicht toleriert und begangenen Fehlern folgen sofort Sanktionen. Typische Items für diese Skala: „Wer A sagt, muß auch B sagen, sonst hätte er von An- fang an ruhig sein sollen“. .,Man kann einem Menschen nicht mehr vertrauen, der schon einmal gelogen hat.“ „Ein gegebenes Versprechen muß man halten, auch wenn es noch so schwer fällt". Analog zur F-Skala und zur A-Skala gab es eine sechsstufige Antwortmöglichkeit.

Zur Erfassung möglicher Unterschiede zwischen Konservatismus und Autoritarismus wurden Skalen eingesetzt, die in unterschiedlicher Weise Anomie messen sollten. Die soziale Desintegration, die mit dem Anomie-Begriff gemeint ist, hat Srole (1956) in einer Skala erfaßt, die u.a. durch mangelndes Vertrauen in die Zukunft, in andere Menschen und insbesondere in Politiker gekennzeichnet ist. Von verschiedenen Autoren wurden Skalen entwickelt, die spezielle Aspekte der Anomie beschrieben. Ellwein/Zoll (1973) haben in einer Faktorenanalyse über Items zu politischem Verhalten Dimensionen entwickelt, die teilweise dem Anomie-Konzept zugeordnet werden können. Mit der Skala „Politische Entfremdung" artikuliert sich die Distanz zur Politik; Politiker und politische Parteien dienen danach nicht dem Volk, sondern nur ihren eigenen Interessen. In der Skala „Politik als Störfaktor" wird die Politik als ein Geschehen angesehen, das den ordnungsgemäßen Gang der Dinge durcheinander bringt und unnötigerweise Probleme schafft. Beide Skalen wurden neben der deutschen Version der SroleSkala (s. Schenk 1979) zur Messung von Anomie eingesetzt.

\subsection{Die Stichproben}

WüStud 69:

Repräsentative Stichprobe Würzburger Studenten 1969 $(\mathrm{N}=350)$. Es handelt sich um eine Zufallsstichprobe, die aus den im Studentensekretariat verfügbaren Adressen gezogen wurde.

EU 70: $\quad$ Studenten, die an einer Eignungsuntersuchung teilnahmen $(\mathrm{N}=66)$; der Fragebogen war nicht Teil der Eignungsuntersuchung, sondern wurde im Anschluß daran freiwillig ausgefüllt.

Krankenpersonal 69: 
$(\mathrm{N}=120)$, die freiwillig an der Untersuchung teilnahmen.

BuWe, I A

BuWe, I B:

BuWe IV

72:

WüStud 75:
Repräsentative Stichprobe von Bundeswehr-Rekruten ( $\mathrm{N}=$ 4 500), denen im Rahmen einer größeren Untersuchung eine Testbatterie vorgelegt wurde, die die erwähnten Skalen enthielt. Die Stichprobe wurde nach Zufall in zwei Untergruppen I A und I B aufgeteilt.

Kleinere Stichprobe von Rekruten $(\mathbf{N}=390)$.

Repräsentative Stichprobe

Würzburger Studenten 1975

$(\mathrm{N}=450)$, analog zu WüStud 69.
TABELLE 1 Korrelation zwischen Konservatismus und Autoritarismus (Spalte 1), zwischen Rigorismus und Konservatismus (Spalte 2) bzw. Rigorismus und Autoritarismus (Spalte 3) und partielle Korrelation zwischen Konservatismus und Autoritarismus unter Ausklammerung von Rigorismus (Spalte 4).

\begin{tabular}{lcccc}
\hline Stichprobe & \multicolumn{5}{c}{ Korrelation } \\
& Kons.-Aut. & Rig.-Kons. & Rig.-Aut. Kons.-Aut.- Rig. \\
\hline EU & .73 & .61 & .71 & .54 \\
Kranken- & & & & \\
pers. & -- & .54 & -- & -- \\
WüStud 69 & -- & .47 & -- & -- \\
BuWe, I A & .67 & .71 & .67 & .37 \\
BuWe, I B & .68 & .73 & .66 & .39 \\
BuWe IV & .57 & -- & -- & -- \\
WüStud 75 & .66 & .65 & .67 & .39 \\
\hline
\end{tabular}

\subsection{Zusammenhang zwischen Konservatismus bzw. Autoritarismus und Rigorismus}

Bevor der Zusammenhang zwischen diesen Variablen dargestellt wird, muß zunächst die Tauglichkeit der Skala „Rigorismus“ überprüft werden. Die Skala hat bei 17 Items eine interne Konsistenz nach Spearman-Brown von .76 (WüStud 69, Krankenpersonal) bzw. bei 16 Items von .87 (BuWe gesamt) und .79 (WüStud 75). Die Skala ist auch bei geringen Itemzahlen reliabel, Beispiel WüStud 75, wo die Skala mit $\mathbf{n}=10$ Items eine Reliabilität von .76 hat. Die Skala ist somit ein sehr verläßliches Testinstrument.

Der Zusammenhang zwischen Konservatismus bzw. Autoritarismus und Rigorismus ist aus Tabelle 1, Spalte 2 und 3 ersichtlich. Es zeigt sich der postulierte positive Zusammenhang zwischen Rigorismus und Autoritarismus bzw. Konservatismus. Die Korrelation von Rigorismus mit den anderen beiden Skalen ist nahezu gleich hoch und recht beachtlich. Es wird somit zwischen 40 und 50 Prozent der Varianz von Rigorismus mit den beiden anderen Skalen erklärt. Konservative bzw. Autoritäre sind danach in einem erheblichen Maße durch eine strikte moralische Haltung gekennzeichnet. Allerdings erklärt der gemeinsame Anteil von Rigorismus mit den anderen beiden Skalen nicht vollständig die Korrelation zwischen Konservatismus und Autoritarismus, wie die partielle Korrelation unter Elimination von Rigorismus zeigt (s. Tab. 1, Spalte 4). Die Korrelationen zwischen

Konservatismus und Autoritarismus gehen je-
Es wird erwartet, daß es einen positiven Zusammenhang zwischen Konservatismus und Autoritarismus gibt. Tabelle 1 zeigt, daß dieser $\mathrm{Zu}$ sammenhang durchgängig hochsignifikant ist und man von einem engen Zusammenhang zwischen Konservatismus und Autoritarismus sprechen kann (s. Tab. 1, Spalte 1). Zur Interpretation dieses Zusammenhangs soll die Skala „Rigorismus" herangezogen werden. 
doch deutlich zurück, wenn man die Variable Rigorismus eliminiert. Besonders augenfällig wird dies, wenn man die Determinationskoeffizienten vergleicht. Während bei der Stichprobe WüStud 75 durch die Korrelation von Konservatismus und Autoritarismus ca. 44 Prozent der Varianz erklärt wird, sinkt dieser Prozentsatz bei Elimination von Rigorismus auf 15 Prozent ab. Rigorismus ist somit als eine sehr wesentliche gemeinsame Variable von Konservatismus und Autoritarismus anzusehen.

\subsection{Wahlverhalten und Konservatismus bzw. Autoritarismus}

Da zwischen Konservatismus und Autoritarismus eine recht enge Verbindung besteht, kann man erwarten, daß beide Variablen in ähnlicher Weise mit Außenkriterien wie z.B. Wahlverhalten korrelieren. Zur Überprüfung dieser Frage wurden Würzburger Studenten (1975) gefragt, welche Partei sie wählen würden, wenn jetzt Bundestagswahlen wären. Zusätzlich wurden sie gefragt, welche Partei sie wählen würden, wenn diese Partei ihre Weltanschauung rein verwirklichen könnte, wobei als Wahlmöglichkeit ,konservativ", ,liberal" und ,sozialistisch" " vorgegeben wurden. Bei den konkreten Parteien wurden die größten aufgeführt (CDU, CSU, FDP, SPD, DKP, KPD, NPD), in die Verrechnung wurden aufgrund der Häufigkeiten nur CDU, CSU, FDP und SPD einbezogen. Getrennt für die beiden Variablen Konservatismus und Autoritarismus

TABELLE 2 Zweifach-Varianzanalysen, getrennt gerechnet für die abhängigen Variablen Konservatismus und Autoritarismus; Stichprobe WüStud 75 (nach Winer 1971).

\begin{tabular}{|c|c|c|c|c|}
\hline Faktor & SS & $\mathrm{df}$ & MS & $p$ von $F$ \\
\hline \multicolumn{5}{|l|}{ Konservatismus } \\
\hline $\begin{array}{l}\text { Weltanschaung } \\
\text { Partei } \\
\text { Weltanschauung } \\
\text { konkrete Partei } \\
\text { Innerhalb Zellen }\end{array}$ & $\begin{array}{c}17.134,22 \\
22.934,98 \\
\\
811,3 \\
133.977,04\end{array}$ & $\begin{array}{l}2 \\
3 \\
6 \\
397\end{array}$ & $\begin{array}{r}8.567,1 \\
7.644,99 \\
\\
135,22 \\
337,47\end{array}$ & $\begin{array}{l}.0001 \\
.0001 \\
.88\end{array}$ \\
\hline \multicolumn{5}{|l|}{ Autoritarismus } \\
\hline $\begin{array}{l}\text { Weltanschauung } \\
\text { Partei } \\
\text { Weltanschauung } \\
\text { konkrete Partei } \\
\text { Innerhalb Zellen }\end{array}$ & $\begin{array}{r}1.813,5 \\
4.679,75 \\
\\
413,13 \\
42.606,65\end{array}$ & $\begin{array}{l}2 \\
3 \\
6 \\
397\end{array}$ & $\begin{array}{r}906,75 \\
1.559,92 \\
\\
68,86 \\
107,32\end{array}$ & $\begin{array}{l}.0005 \\
.0001 \\
.70\end{array}$ \\
\hline
\end{tabular}

wurden zwei Zweifach-Varianzanalysen (mit den Faktoren ,konkrete Partei“" und ,reine Weltanschauung") gerechnet. Das Ergebnis beider Varianzanalysen ist aus Tab. 2 ersichtlich.

Bei beiden Varianzanalysen ergibt sich, daß die beiden Variablen ,Weltanschauung“ und „konkrete Partei" signifikant sind, es jedoch keinen Interaktionseffekt gibt. Wahlpräferenzen lassen sich danach sowohl durch die konservative wie durch die autoritäre Einstellung in gleicher Weise differenzieren. Die Reihung der Parteien bzw. der Weltanschauungen erfolgt durch die beiden Einstellungen in der gleichen Weise. Beispielhaft werden die Mittelwerte für die Weltanschauungen in Graphik 1 dargestellt. Danach hat sowohl bei Konservatismus wie bei Autoritarismus die konservative Richtung den höchsten Wert, gefolgt von den Liberalen und klar abgesetzt davon der sozialistischen Richtung.

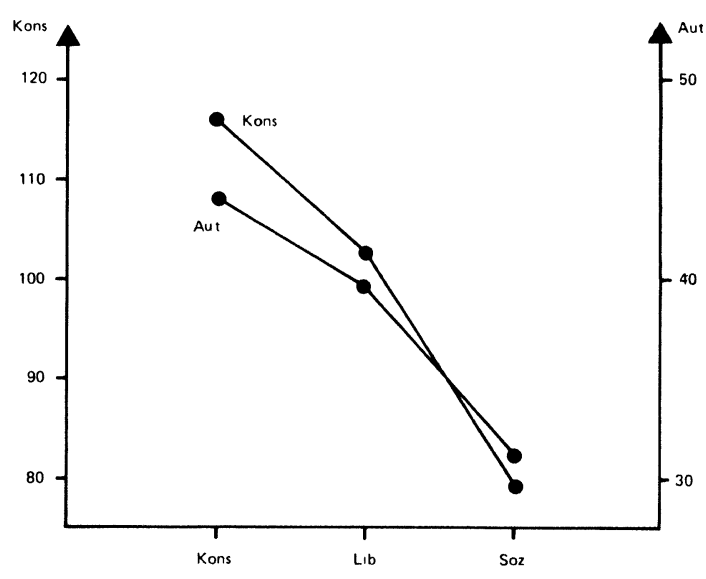

GRAPHIK I Mittelwerte für Weltanschauungsgruppen, getrennt für Konservatismus und Autoritarismus; WüStud 75

\subsection{Beziehung von Autoritarismus und Kon- servatismus zu Anomie.}

Die bisherigen Ergebnisse sprechen für einen engen Zusammenhang $z$ wischen Konservatismus und Autoritarismus. Wäre man bereit, den Rest der fehlenden Korrelation zwischen Konservatismus und Autoritarismus als Fehlervarianz abzutun, so könnte man beide Konzepte als identisch betrachten. Es wurde jedoch vermutet, daß es neben den Gemeinsamkeiten auch Unterschiede zwischen Konservatismus und Autoritarismus 
TABELLE 3 Korrelation von Konservatismus bzw. Autoritarismus mit „Politik als Störfaktor“ (= POST), „Politische Entfremdung“" (= POEN) und „Anomie nach Srole" (= ANS)

Stichprobe

Korrelation

POST-Aut. POST-Kons. POEN-Aut. POEN-Kons. ANS-Aut ANS-Kons

\begin{tabular}{lllllll}
\hline BuWe, I A & .48 & .29 & .51 & .27 & -- & -- \\
BuWe, I B & .47 & .27 & .45 & .19 & .54 & .29 \\
BuWe IV & .51 & .29 & .50 & .20 & -- & -- \\
WüStud 75 & .43 & .19 & .23 & -.21 & .24 & -.13 \\
\hline
\end{tabular}

gibt, die dann für die Dämpfung der Korrelation verantwortlich sein könnten. Hier wurde an die verschiedenen Formen der Anomie gedacht, deren Zusammenhänge mit Konservatismus bzw. Autoritarismus aus Tab. 3 ersichtlich sind.

Aus Tabelle 3 sind deutliche Unterschiede zwischen Autoritarismus und Konservatismus erkennbar. Bei den verschiedenen Stichproben von Rekruten korrelieren die Anomie-Skalen deutlich höher mit Autoritarismus als mit Konservatismus. Dies wird besonders deutlich, wenn man den Determinationskoeffizienten für die gefundenen Korrelationen berechnet. In der Stichprobe BuWe IA wird durch die Korrelation von POEN und Aut. ca. 25 Prozent der Varianz erklärt, durch die Korrelation von POEN mit Kons. hingegen nur 7 Prozent der Varianz. Der Zusammenhang von Autoritarismus mit Anomie ist somit für diese Stichproben weit enger als der für Konservatismus mit Anomie.

Klarer werden die Unterschiede zwischen Konservatismus und Autoritarismus für die Stichprobe WüStud 75, eine Stichprobe, die in der sozialen Schichtung einen höheren Rang hat. Hier findet sich für die Variable „Politik als Störfaktor" zwar der übliche Unterschied in der Höhe der Korrelation, bei den eindeutiger Anomie messenden Skalen „Politische Entfremdung" und „Anomie nach Srole“ aber wird die Korrelation gegenläufig. Autoritäre sind danach eher anomisch, Konservative sind eher wenig anomisch. Die Korrelationen selbst sind nicht allzu hoch, bedeutsam ist hier wohl die Umkehrung des Vorzeichens. Danach kann man sagen, daß Konservatismus und Autoritarismus - definiert durch die vorliegenden Skalen - nicht völlig identisch sind. Konservatismus hat im Vergleich zu Autoritarismus einen geringeren Zusammenhang mit Anomie, bei einer bildungsmäßig höhe- ren Schicht ist die Beziehung von Autoritarismus mit Anomie bzw. Konservatismus mit Anomie sogar konträr. Die Gleichsetzung von Konservatismus und Autoritarismus erscheint danach nicht gerechtfertigt.

\section{Zusammenfassung}

Es konnte gezeigt werden, daß Konservatismus und Autoritarismus hoch miteinander korrelieren. Diese Korrelation kann größtenteils durch eine dritte Variable, eine rigorose moralische Einstellung erklärt werden. Allerdings wi.d durch die Variable Rigorismus die Korrelation zwischen Konservatismus und Autoritarismus nicht vollständig erklärt.

Die Korrelation zwischen diesen beiden Variablen spricht für eine weitgehende Gleichsetzung dieser beiden Einstellungen, was durch die Beziehung zum Wahlverhalten untermauert wird. Konservatismus und Autoritarismus differenzieren in gleicher Weise Wahlpräferenzen. Gegen eine solche Gleichsetzung beider Einstellungen spricht jedoch, daß Konservatismus weniger stark mit Anomie korreliert, als dies Autoritarismus tut. Bei bildungsmäßig höheren Schichten korreliert Anomie sogar negativ mit Konservatismus. Konservatismus sollte nach den vorliegenden Ergebnissen nicht mit Autoritarismus gleichgesetzt werden, das Verfehlen einer sehr hohen Korrelation ist nicht allein auf Fehlervarianz, sondern auch auf signifikante Unterschiede zurückzuführen.

Die Untersuchung zeigt, daß es Gemeinsamkeiten und Unterschiede zwischen Konservatismus und Autoritarismus gibt. Es besteht die Gefahr, daß durch eine vergröberte Messung von Konservatismus - wie sie von Wilson/Patterson (1968) und Siddiqui/Jansen/Haara (1971) vorge- 
nommen wurde - mehr diese Gemeinsamkeiten und weniger die Unterschiede gesehen werden. Die hier gefundenen Korrelationen zwischen Konservatismus und Autoritarismus wird man auch aufgrund der gegenwärtigen operationalisierten Fassung von Konservatismus sehen müssen. Es ist möglich, daß bei dem Versuch der Konstruktion reliabler Skalen für die konservative Einstellung durch die Notwendigkeit, kurze und prägnante Items zu formulieren, eine Annäherung von Konservatismus und Autoritarismus erfolgte, die durch die gewählte Methode bedingt ist. Eine Inspektion bestehender Skalen zeigt überdies, daß durch sie das Spektrum der konservativen Einstellung nicht hinreichend repräsentiert wird. Es muß sich zeigen, ob eine noch stärker am Konzept des Konservatismus erfolgte Operationalisierung von Konservatismus die gleichen Zusammenhänge erbringt. Die gegenwärtigen Ergebnisse sprechen für wesentliche Gemeinsamkeiten zwischen beiden Einstellungen, allerdings mit Akzentunterschieden, so daß sich eine Gleichsetzung verbietet. Eine präzisere Fassung von Konservatismus via Skala könnte die Unterschiede zwischen beiden Einstellungen möglicherweise deutlicher werden lassen.

\section{Literatur:}

Adorno, T.W./Frenkel-Brunswik, E./Levinson, D.J./Sanford, R.N., 1950: The authoritarian personality.

New York: Harper

Burke, E., 1967: Betrachtungen über die französische Revolution. dt. Ausgabe Frankfurt: Suhrkamp

Carlson, H.B., 1934: Attitudes of undergraduate students. Journal of social psychology 5: 202-212

Ellwein, T./Zoll, R., 1973: Politische Bildung und empirische Sozialforschung. Materialien zur politischen Bildung 1: 34-55.

Eysenck, H.J., 1954: Psychology of politics. London: Routledge and Kegan Paul.

Eyseneck, H.J., 1975: The structure of social attitudes. British Journal of Social and Clinical Psychology 14: $323-331$
Ferguson, L.W., 1939: Primary social attitudes. Journal of psychology 8: 217-223

Freyhold, M.v., 1971: Autoritarismus und politische Apathie. Frankfurt.

Huntington, S.P., 1957: Conservatism as an ideology. American Political and Social Science Review 51.

Kohn, P.M., 1972: The authoritarianism-rebellion scale: A balanced $F$ scale with left-wing reversals. Sociometry 35: 177-189.

Kohn, P.M., 1974: Authoritarianism, rebelliousness, and their correlates among British undergraduates. British Journal of Social and Clinical Psychology 13: $245-255$.

McDill, E.L., 1961: Anomie, authoritarianism, prejudice and socio-economic status. Social Forces 39: 239-245.

Mühlenfeld, H., 1952: Politik ohne Wunschbilder. München.

Rausche, A., 1979: PSYST-200. Programmsystem zur Datenanalyse in der Psychologie. Würzburg, unveröffentlicht.

Ray, J.J., 1973: Conservatism, authoritarianism and related variables. In: G.D. Wilson (Hrsg.): The psychology of conservatism. London: Academic press.

Roberts, A.H./Rokeach, M., 1956: Anomie, authoritarianism, and prejudice. American Journal of Sociology 61 .

Roghmann, K., 1966: Dogmatismus und Autoritarismus. Meisenheim: Hain.

Rokeach, M., 1960: The open and closed mind. New York: Basic books.

Schenk, J., 1979: Persönlichkeit des Drogenkonsumenten. Göttingen: Hogrefe.

Schenk, J., 1980: Konservatismus und Klerikalismus. Psychologische Beiträge 21: 145-162.

Schneider, J./Minkmar, H., 1972: Deutsche Neukonstruktion einer Konservatismus-Skala. Diagnostica 18.

Siddiqui, J.A./Jansen, R./Haara, A., 1971: Konservatismus: Eindimensional oder mehrdimensional? Psychologische Beiträge 13: 26-37.

Srole, L., 1956: Social integration and certain correllaries. Sociological Review 21.

Struening, E.L./Richardson, A.H., 1965: A factor analytic exploration of the alienation, anomia, and authoritarianism domain. American Sociological Review 30: 768-774.

Thomas, D.R., 1975: Conservatism, authoritarianism and child-rearing practices. British Journal of Social and Clinical Psychology 14: 97-98.

Wilson, G.D./Patterson, J.R., 1968: A new measure of conservatism. British Journal of Social and Clinical Psychology 7: 264-269.

Winer, B.J., 1971: Statistical principles in experimental design. New York: McGraw Hill, 2. Auflage. 